\title{
Tecnura
}

\section{Impacto ambiental del vertimiento de aguas servidas en aglomerados urbanos ilegales del municipio de Villavicencio, Colombia}

\section{Environmental Impact of Sewage Discharge Into Illegal Urban Agglomerates in Villavicencio, Colombia}

\author{
Álvaro Javier Moyano Salcedo (iD) 1 , Fabián Dario Cuadros Segura (iD) 2 Adrián Mateo Pabón \\ Laverde ${ }^{(i D}$, Jassbleydi Valentina Trujillo Arias (iD 4
}

Fecha de Recepción: 05 de Septiembre de 2020

Fecha de Aceptación: 24 de Febrero de 2021

Cómo citar: Moyano-Salcedo., A.J. Cuadros-Segura., F.D. Pabón-Laverde., A.M. y Trujillo-Arias., J.V. (2021). Impacto ambiental del vertimiento de aguas servidas enaglomerados urbanos ilegales del municipio de Villavicencio, Colombia. Tecnura, 25(68), 43-62. https: / / doi.org/10.14483/22487638.16273

\section{Resumen}

Contexto: La conformación de conglomerados habitacionales ilegales, en la mayoría de los casos, sin saneamiento básico adecuado, puede generar deterioro de los recursos naturales. El presente estudio evaluó el impacto ambiental asociado al vertimiento de aguas servidas urbanas (ASU) sobre suelos y cuerpos de agua, en un aglomerado urbano ilegal del municipio de Villavicencio.

Metodología: A partir de 15 puntos de muestreo se determinaron parámetros fisicoquímicos de agua: serie de sólidos, turbiedad, color, demanda química de oxígeno (DQO), $\mathrm{pH}$, conductividad eléctrica (CE), oxígeno disuelto (OD); y de suelo: $\mathrm{pH}, \mathrm{CE}$, capacidad de intercambio catiónico (CIC) y concentración de $\mathrm{C}, \mathrm{N}$ y elementos traza, para identificar impactos sobre los recursos en el área de estudio.

Resultados: Las muestras de ASU presentaron valores elevados en parámetros como turbiedad (326 NTU), color (744 UPC) y DQO bruta (600 a $1500 \mathrm{mg} \mathrm{l}^{-1}$ ). En los análisis de cuerpos de agua aledaños al sector, la DQO varió entre 368 y 429 mg $1^{-1}$. Asimismo, el OD osciló entre 5,7 y 7,1 $\mathrm{mg}^{-1}$, valores que resultaron favorables para los cauces; sin embargo, fueron bajos para el ASU $\left(0,9 \mathrm{mg} \mathrm{l}^{-1}\right)$. Por su parte, se determinó una alteración significativa en propiedades fisicoquímicas del suelo como pH (5,8-8,2), CIC (8,2-16,78 mEq-g $\left.100 \mathrm{~g}^{-1}\right), \mathrm{CE}\left(57,6-517,3 \mu \mathrm{S} \mathrm{cm}^{-1}\right)$ y N (0,03-0,84 mg kg $\left.{ }^{-1}\right)$ asociadas principalmente a los vertimientos.

\footnotetext{
${ }^{1}$ Ingeniero ambiental, especialista en Gestión Ambiental, magíster en Gestión del Agua, magíster en Ciencia y Tecnología Ambiental. Coordinador grupo de investigación Geoamenzas e Ingenieria Civil. Docente tiempo completo, Universidad Santo Tomás Villavicencio, sede Aguas Claras, Colombia. Contacto: alvaromoyano@usantotomas.edu.co

${ }^{2}$ Biólogo, magíster en Ciencias Microbiológicas y candidato a doctor en Biotecnología. Profesor agregado, Laboratorio de Microbiología, Instituto de Biología, Pontificia Universidad Católica de Valparaíso, Valparaíso, Chile. Contacto: fabian.cuadros.s@mail.pucv.cl

${ }^{3}$ Ingeniero civil, Universidad Santo Tomás, Villavicencio, sede Aguas Claras, Colombia. Contacto: adrianpabon@usantotomas.edu.co

${ }^{4}$ Ingeniera civil, Universidad Santo Tomás, Villavicencio, sede Águas Claras, Colombia. Contacto: jassbleyditruji1lo@usantotomas.edu.co
} 
Impacto ambiental del vertimiento de aguas servidas en aglomerados urbanos ilegales del municipio de Villavicencio, Colombia

Moyano-Salcedo., A.J. Cuadros-Segura., F.D. Pabón-Laverde., A.M. y Trujillo-Arias., J.V.

Conclusiones: Fue posible identificar un efecto negativo en los recursos agua y suelo, generado por descargas descontroladas de ASU en el lugar, lo cual se encuentra relacionado una pérdida de servicios ambientales y baja calidad de vida.

Palabras clave:agua servida urbana, aglomerados urbanos ilegales, calidad del agua, calidad del suelo, impacto ambiental.

\begin{abstract}
Context: The formation of illegal housing conglomerates, in most cases, without adequate basic sanitation, can lead to deterioration of natural resources. The aim of this study was to evaluate the environmental impact associated with the urban sewage discharge (USD) on ground and water bodies in an illegal urban agglomeration in the Villavicencio municipality. Methodology: From 15 sampling points, physicochemical parameters of water were determined: solids series, turbidity, color, chemical oxygen demand (COD), $\mathrm{pH}$, electrical conductivity (EC), dissolved oxygen (DO), and soil: $\mathrm{pH}, \mathrm{EC}$, cation exchange capacity (CEC) and $\mathrm{C}, \mathrm{N}$ and trace elements concentration, to relate and identify the impacts on resources in the study area.

Results: The USD samples presented high values in parameters such as turbidity (326 NTU), color (744 UPC) and crude COD that ranged from 600 to $1500 \mathrm{mg} \mathrm{L}^{-1}$. In the water bodies surrounding the sector, the COD varied between 368 and $429 \mathrm{mg} \mathrm{L}^{-1}$. Likewise, DO ranged from 5,7 to $7,1 \mathrm{mg} \mathrm{L}^{-1}$, values that were favorable for the channels, however, they were low for the USD $\left(0,9 \mathrm{mg} \mathrm{L}^{-1}\right)$. On the other hand, a significant alteration was determined in soil physicochemical properties such as pH (5,8-8,2), CEC (8,2-16,8 mEq-g $\left.100 \mathrm{~g}^{-1}\right)$, EC (57,6- 517,3 $\left.\mu \mathrm{S} \mathrm{cm}^{-1}\right)$ and $\mathrm{N}\left(0,03-0,84 \mathrm{mg} \mathrm{Kg}^{-1}\right)$ mainly associated with discharges.

Conclusions: It was possible to identify a negative effect on water and soil resources, generated by uncontrolled discharges of USD in the place, which is related to a loss of environmental services and low life quality.
\end{abstract}

Keywords: urban sewage illegal urban agglomerates, water quality, soil quality, environmental impact.

\title{
Tabla de Contenidos
}

METODOLOGÍA $\quad 46$

Características ambientales y puntos de muestro seleccionados . . . . . . . . . . . . . 46

Toma de muestras y análisis de la calidad de agua residual . . . . . . . . . . . . . . . . . . . . . . . . . . . .

Toma y análisis de muestra de suelo . . . . . . . . . . . . . . . . . . . 47

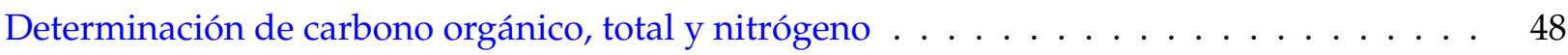

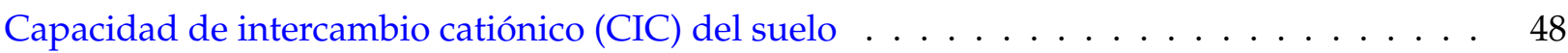

Análisis estadístico . . . . . . . . . . . . . . . . . . . . . . . . 49

$\begin{array}{lr}\text { RESULTADOS } & 49\end{array}$

Calidad del agua $\ldots \ldots \ldots \ldots \ldots \ldots \ldots \ldots \ldots \ldots \ldots$

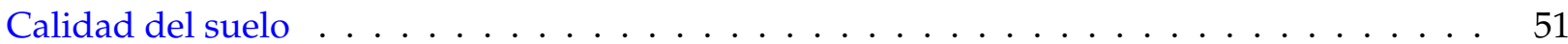




\section{INTRODUCCIÓN}

El aumento continuo de la población y actividades productivas llevan a la expansión de los municipios. En Colombia, este crecimiento en algunos casos se da de forma desordenada, sin acompañamiento de entes de planeación; por esto, los nuevos aglomerados urbanos carecen, en su mayoría, de sistemas de saneamiento básico. Además, como base económica, gran parte de estas comunidades desarrollan actividades mayormente pecuarias (porcicultura y avicultura) para su subsistencia (Hernández-Gómez, Rojas-Robles y Sánchez-Calderón, 2013). Como consecuencia, existe un aumento en la demanda de agua que origina una presión en áreas donde hay escasez o dificultad para su obtención; además, el desarrollo de estas actividades genera aguas servidas que son vertidas sin tratamiento y pueden ocasionar impactos ambientales y a la salud humana.

Dependiendo del origen, las aguas servidas poseen diferentes tipos y cantidades de contaminantes, entre los cuales se destacan la concentración de materia orgánica (MO), nutrientes, sólidos y químicos; por lo que su estudio, tratamiento y remoción es un gran desafío en la actualidad (Subtil, Moyano y Bez, 2018). Las aguas servidas domiciliaras (ASD) son aquellas procedentes de las descargas de los retretes, servicios sanitarios, sistemas de aseo personal, áreas de cocinas y lavado de elementos de aseo (Subtil, Moyano y Bez, 2018) y las de origen pecuario son generadas de la limpieza de corrales, establos, cocheras, etc., lo cual involucra mayormente el arrastre de las heces fecales de los animales y los sobrantes alimenticios. Debido a esto, y a los impactos que dichas aguas pueden causar, las autoridades ambientales tienen establecidos parámetros de vertimiento (Resolución 0631 del 2015 en Colombia) (Rodríguez-Miranda, García-Ubaque y Pardo-Pinzón, 2015) que deben ser cumplidos para evitar sanciones y multas, lo que pertinente estudiar y proponer sistemas de tratamiento eficientes para la depuración de las aguas servidas.

La remoción convencional de contaminantes del agua se puede ejecutar por medio de sistemas físicos, químicos y biológicos. En el caso de viviendas rurales o unifamiliares, una solución útil ha sido la implementación de pozos sépticos como sistema biológico anaerobio de tratamiento, que pueden definirse como un depósito que propicia la sedimentación de sólidos y los retiene durante un tiempo para facilitar el crecimiento de microorganismos que degradan la MO (Londoño et al., 2002, Lampoglia, Agüero y Barrios, 2008).

El presente estudio se desarrolló en el aglomerado urbano ilegal El Brillante, ubicado en la comuna 5 del municipio de Villavicencio, Meta (Colombia). Su conformación partió en el año 2013, promovido por invasión de predios rurales por pobladores locales de una zona denominada Fondo 
Ganadero. Este lugar no cuenta con red de alcantarillado y como solución para el almacenamiento temporal de las aguas servidas urbanas (ASU), se han construido pozos sépticos. Sin embargo, el inadecuado diseño, operación y mantenimiento, así como su vertimiento directo a sectores aledaños y al río Ocoa (utilizado con fines recreativos), hacen necesario el estudio del impacto ambiental en las fuentes hídricas y suelo asociado.

\section{METODOLOGÍA}

\section{Características ambientales y puntos de muestro seleccionados}

El sector El Brillante, del municipio de Villavicencio, se encuentra a 423 m s.n.m. en el piedemonte llanero del departamento del Meta (Colombia). Presenta una humedad relativa promedio anual de $76 \%$, evapotranspiración potencial hasta de $1,305 \mathrm{~mm}$, con temperatura promedio de $25,5{ }^{\circ} \mathrm{C}$ con épocas templadas (abril a octubre) y calurosas (diciembre a marzo); su precipitación media anual es de 321,3 mm, siendo mínima en el mes de enero (51 mm) y máxima en mayo (529 mm) (Ideam, 2015). En temporada de invierno, los cuerpos de agua pueden presentar caudales máximos y mayor poder de dilución de contaminantes, es decir, probablemente el impacto ambiental asociado al vertimiento de aguas servidas domésticas en esta época sea menor, y en periodos de invierno pueden existir quebradas intermitentes, lo que promueve el transporte de contaminantes.

En la figura 1 se presentan los puntos de muestreo (agua y suelo) seleccionados y las fuentes hídricas aledañas en donde se realizan los vertimientos puntuales. El principal afluente cercano al aglomerado urbano el brillante es el río Ocoa y en invierno hace presencia una quebrada intermitente que atraviesa la zona por el costado oeste. El suelo característico de la zona de estudio está determinado por depósitos y llanuras aluviales de edad cuaternaria (Ideam, 2015), constituidos por acumulación del río Ocoa y del caño Campo Alegre presente en el área de estudio.

En cuanto a biodiversidad e importancia ambiental, el piedemonte llanero colombiano es una franja con abundancia de biomasa y de ecosistemas estratégicos (Briceño, 2015). Su valor estratégico radica en que comprende una zona de transición ecológica entre bosques de montaña y sabanas de altillanura, abastecida por cauces hídricos de drenaje de la vertiente oriental de la cordillera que generan suelos sedimentarios de mayor fertilidad a la de aquellos que se encuentran Orinoquía adentro (Stevenson, Ramírez, Casas y Henao-Díaz, 2017,IGAC, 2006). Así, en el área de estudio confluyen especies típicas de estos dos ecosistemas en mención, las cuales son susceptibles al impacto generado por el inadecuado manejo y gestión de las aguas urbanas vertidas sin tratamiento previo.

\section{Toma de muestras y análisis de la calidad de agua residual}

Fueron seleccionados 15 puntos de muestreo según el método estadístico de estratificación óptima (Cantanhede, Monge, Sandoval y Caycho, 2005), tomando un nivel de confianza del $95 \%$. Los 


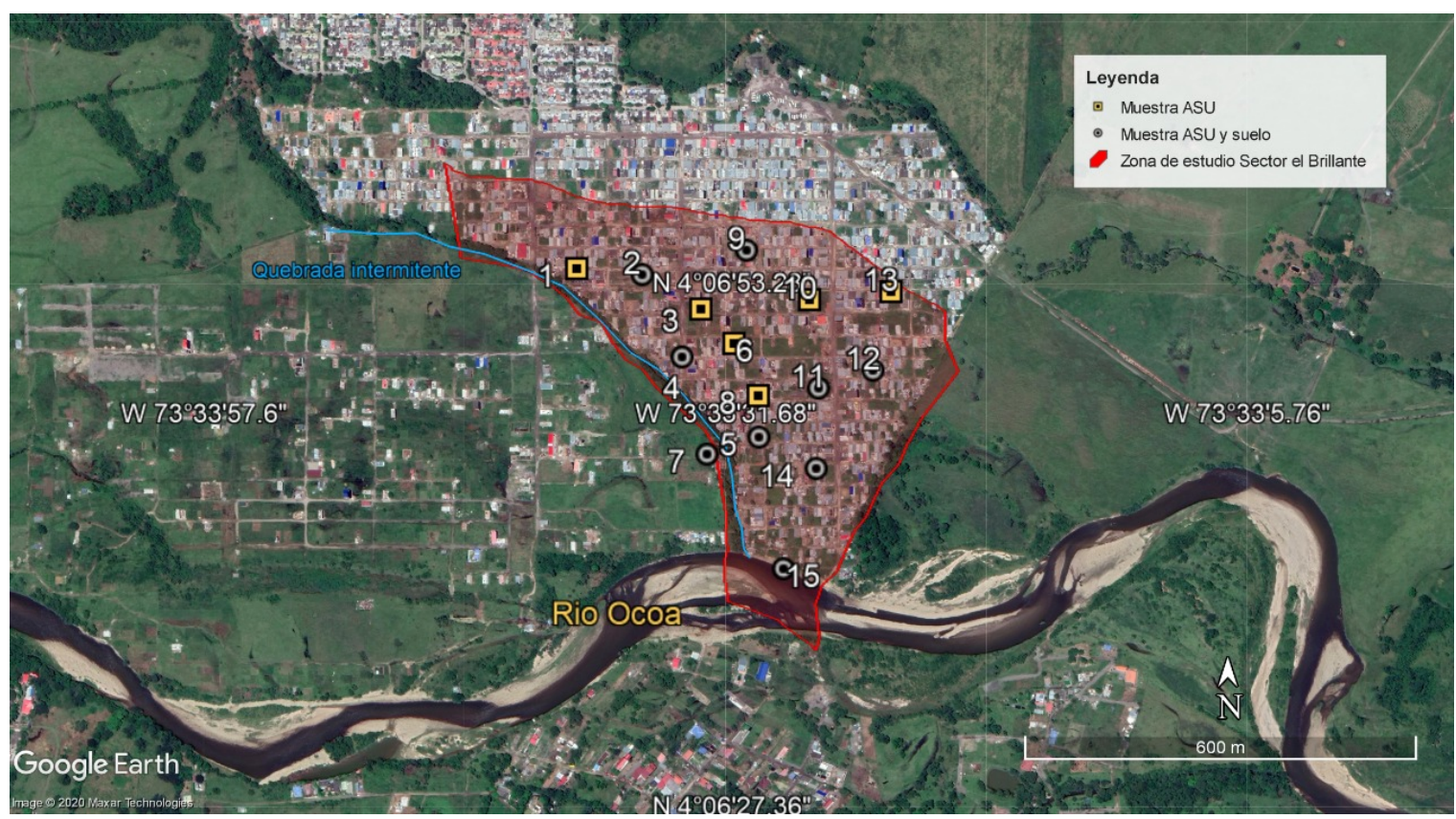

Figura 1. Cuerpos hídricos aledaños al área de investigación

Fuente: elaboración propia, adaptado de Google Maps.

puntos correspondieron a la salida directa de ASU de tuberías usadas para su conducción, retiro directo de los pozos sépticos a 5 y $10 \mathrm{~m}$ del vertimiento puntual a las fuentes hídricas (quebrada intermitente y río Ocoa) (figura 1).

La toma de muestra en los cauces de agua se realizó con el fin de estimar la contaminación asociada. Para el análisis de la calidad del agua servida, las muestras se recogieron de los puntos de vertimiento final de forma semanal durante tres meses y se determinaron por duplicado los principales parámetros de calidad del agua, serie de sólidos y DQO (Kit COD USEPA, Hatch) (tabla I). La metodología de análisis usada fue la descrita por (Rice, Baird e Eaton, 2017).

\section{Toma y análisis de muestra de suelo}

Se tomaron muestras compuestas de la capa superficial del suelo $(20 \mathrm{~cm})$ en nueve puntos influenciados por ASU (figura 1), utilizando el método de barreno bajo los parámetros descritos por la (USDA, 2014). Las muestras fueron secadas a $40{ }^{\circ} \mathrm{C}$ por $48 \mathrm{~h}$, maceradas y tamizadas a $500 \mu \mathrm{m}$, debido a que estas presentan visualmente material inorgánico constituido principalmente por partículas finas inferiores a esta medida (Sadzawka et al., 2006). La humedad del suelo fue calculada por triplicado a partir de la diferencia de pesos. A cada muestra se le determinó por triplicado el pH y la conductividad eléctrica por métodos de rutina (USDA, 2014). Se seleccionó el punto 2, por ser de mayor interés al mantenerse afectado por la constante irrigación de ASU, a la cual se le determinó fós- 
Impacto ambiental del vertimiento de aguas servidas en aglomerados urbanos ilegales del municipio de Villavicencio, Colombia

Moyano-Salcedo., A.J. Cuadros-Segura., F.D. Pabón-Laverde., A.M. y Trujillo-Arias., J.V.

Tabla I. Programa de monitoreo sector El Brillante

\begin{tabular}{cccc}
\hline Análisis & Unidad & Frecuencia de muestreo & Método \\
\hline Temperatura & ${ }^{\circ} \mathrm{C}$ & 5 muestras/semana & - \\
\hline $\mathrm{OD}$ & $\mathrm{mgO2} 1^{-1}$ & - & - \\
\hline $\mathrm{pH}$ & - & - & $2320-\mathrm{B}$ \\
\hline Conductividad & $\mathrm{Bar}$ & - & - \\
\hline Color & $\mathrm{UPC}$ & - & - \\
\hline Turbiedad & $\mathrm{UNT}$ & 5 muestras/semana & 2130 \\
\hline DQO & $\mathrm{mg} \mathrm{l^{-1 }}$ & 3 muestra/semana & 8000 \\
\hline Serie de sólidos (ST, SSV) & $\mathrm{mg} \mathrm{l}^{-1}$ & 4 muestras/semana & 2540 \\
\hline
\end{tabular}

ST: sólidos totales, SSV: sólidos suspendidos volátiles.

Fuente: elaboración propia.

foro disponible por método de flujo continuo con extracción de Bray II y concentración de elementos traza (Al, As, Ba, B, Cu, Cr, Sr, Li, Mn, Ni, Pb, V y Zn), mediante digestión ácida asistida con microondas (US EPA, 2007) y determinada con espectroscopia de plasma acoplado inductivamente (US EPA, 1994).

\section{Determinación de carbono orgánico, total y nitrógeno}

La concentración de carbono total se calculó por método gravimétrico a partir de $1 \mathrm{~g}$ de suelo seco por triplicado, el cual fue sometido a calcinación a $350{ }^{\circ} \mathrm{C}$ por $6 \mathrm{~h}$ y $505{ }^{\circ} \mathrm{C}$ por $6 \mathrm{~h}$ (Miyazawa et al., 2000). El porcentaje de MO se calculó por método de Walkley Black (Enang, Yerima, Kome y Van Ranst, 2018), utilizando $\mathrm{Fe}\left(\mathrm{NH}_{4}\right)_{2}\left(\mathrm{SO}_{4}\right)_{2} 1 \mathrm{~N}$ en medio ácido como agente titulante. Para la concentración de nitrógeno orgánico se utilizó destilación Kjeldhal (Bradstreet, 1954) con algunas modificaciones (Amin y Flowers, 2004). Para esto, se tomó $1 \mathrm{~g}$ de muestra tratada, que fue digerida con $\mathrm{H}_{2} \mathrm{SO}_{4}$ concentrado y tableta Kjeldahl de digestión para nitrógeno Missouri (4,98 g K $\mathrm{SO}_{4}$ y 0,02 $\left.\mathrm{g} \mathrm{CuSO}_{4} 7 \mathrm{H}_{2} \mathrm{O}\right)$ a $350{ }^{\circ} \mathrm{C}$ por $6 \mathrm{~h}$. Pasado este tiempo, se ajustó el $\mathrm{pH}$ con $\mathrm{H}_{2} \mathrm{Odd}$ y $\mathrm{NaOH} 32 \%$. La muestra fue tratada en destilador Kjendhal (BÜCHI Distillation Unit K-355) por 5 min y el producto rescatado fue diluido en $\mathrm{H}_{3} \mathrm{BO}_{3} 2 \%$. Se realizó titulación utilizando $\mathrm{H}_{2} \mathrm{SO}_{4} 0,02 \mathrm{~N}$ e indicador mixto (Rojo de metilo-Azul de metileno).

\section{Capacidad de intercambio catiónico (CIC) del suelo}

Para determinar la CIC se elaboró una solución de suelo seco y $\mathrm{NH}_{4} \mathrm{CH}_{3} \mathrm{CO}_{2} 1 \mathrm{M}$ 1:5 p/v por $24 \mathrm{~h}$ en agitación fuerte. Pasado este tiempo, se centrifugó a 5000 g por 5 min, se descartó el sobrenadante y 
el precipitado fue lavado en agitación con $10 \mathrm{ml}$ de etanol absoluto por $5 \mathrm{~min}$. Se realizó nuevamente centrifugado y al pellet final se le agregaron $10 \mathrm{ml}$ de $\mathrm{NaCl} 10 \%$ y se agitó fuertemente por $5 \mathrm{~min}$; se rescató el sobrenadante y se repitió este proceso dos veces para un volumen final de $30 \mathrm{ml}$. A la solución se le añadieron $10 \mathrm{ml}$ de formaldehido $38 \%$, se agitó y tituló con $\mathrm{NaOH}$ 0,1 M utilizando fenolftaleína como indicador.

\section{Análisis estadístico}

Con el fin de determinar la relación y el comportamiento entre las diferentes variables halladas en este estudio, se adelantó un análisis de regresión lineal y regresión múltiple para observar los comportamientos de las variables. De igual forma, para describir en conjunto la relación y relevancia de estas, se aplicó un modelo de análisis de componentes principales utilizando el software Minitab $19 \circledR$.

\section{RESULTADOS}

\section{Calidad del agua}

Todos los parámetros presentan valores elevados en ASU bruta y valores más bajos en las fuentes hídricas, dependiendo su potencial de dilución. En el caso del río Ocoa, fue posible observar los valores más elevados (tabla II), debido a que se encuentran directamente influenciados por aportes de aguas contaminadas; sin embargo, los resultados muestran una elevada contaminación en los puntos de muestreo de ambas fuentes ( 5 a $10 \mathrm{~m}$ de un punto de vertimiento de ASU), lo que puede generar un impacto ambiental, afectando a la salud de los habitantes. Asimismo, es importante resaltar que las características del agua servida bruta están por encima de los valores típicos reportados (DQO: 400-800 mg $~^{-1}$ ) para clasificarla como ASD (Subtil, Moyano y Bez, 2018, Ayaz, Aktaş Akça y Findik, 2015). Este aumento puede deberse a aportes de actividades pecuarias a pequeña escala evidenciadas en el sector.

Las elevadas temperaturas medidas y las variaciones de $\mathrm{pH}$ muestran que existen condiciones ambientales desfavorables para la subsistencia de la diversidad. Por otra parte, la turbiedad (326 NTU) y el color (744 UPC) encontrados en el ASU bruta fueron propios de aguas muy contaminadas; esto indica la existencia de aportes por procesos extractivos o erosivos, y en este caso, la posibilidad de estar fuertemente relacionado a la contaminación orgánica (elevado aporte de DQO, DBO, amonio, etc.) (Samboni, Aldemar y Yesid, 2011, Torres et al., 2010, Madsen, Lévêque, Omiste y Miyagi, 2018). La conductividad, ST y SSV tuvieron valores ligeramente por encima de los rangos normales de ASU, los cuales son limitantes para algunos usos y, desde el punto de vista limnológico, presentan condiciones desfavorables para el establecimiento del ecosistema hídrico. 
Impacto ambiental del vertimiento de aguas servidas en aglomerados urbanos ilegales del municipio de Villavicencio, Colombia

Moyano-Salcedo., A.J. Cuadros-Segura., F.D. Pabón-Laverde., A.M. y Trujillo-Arias., J.V.

Tabla II. Parámetros de calidad del ASU bruta del río Ocoa y la quebrada intermitente (QI) del sector El Brillante

\begin{tabular}{cccccc}
\hline Parámetros & Unidades & ASD bruta & Río Ocoa & QI & $\begin{array}{c}\text { Valores máximos } \\
\text { de vertimiento ASD* }\end{array}$ \\
\hline $\mathrm{pH}$ & - & 7,5 & 6,6 & 6,7 & $6-9$ \\
\hline Turbiedad & $\mathrm{NTU}$ & $326 \pm 166$ & $48 \pm 26$ & $26 \pm 7$ & 2 \\
\hline Color & $\mathrm{UPC}$ & $744 \pm 356$ & $31 \pm 13$ & $21 \pm 10$ & 15 \\
\hline $\mathrm{CE}$ & $\mathrm{MS} \mathrm{cm}^{-1}$ & $524 \pm 282$ & $128 \pm 75$ & $111 \pm 58$ & 1000 \\
\hline $\mathrm{ST}$ & $\mathrm{mg} \mathrm{l}^{-1}$ & $873 \pm 224$ & - & - & 720 \\
\hline $\mathrm{SSV}$ & $\mathrm{mg} \mathrm{l}^{-1}$ & $488 \pm 202$ & - & - & 300 \\
\hline
\end{tabular}

CE: conductividad eléctrica, ST: sólidos totales, SSV: sólidos suspendidos volátiles, OD: oxígeno disuelto, DQO: demanda química de oxígeno.

Fuente: elaboración propia. $\left(^{*}\right)$ Basado en Resolución 2115/2007 y (Madsen, Lévêque, Omiste y

$$
\text { Miyagi, 2018). }
$$

La DQO medida en el agua presenta una elevada contaminación orgánica, típica de industrias agroalimentarias, como lácteos o explotaciones pecuarias (Sarkar, Chakrabarti, Vijaykumary Kale, 2006, Makara y Kowalski, 2015, Cecconet, Molognoni, Callegari y Capodaglio, 2018), pues es posible observar estudios para aguas asociadas a actividades de sacrificio porcino y bovino en donde se han reportado valores elevados de DQO (1200 mg $\left.\mathrm{l}^{-1}\right)$, DBO (5000 $\left.\mathrm{mg} \mathrm{l}^{-1}\right)$ y SST (1500 y $3500 \mathrm{mg}$ $1^{-1}$ ) (Hernández y Sánchez, 2015,Pérez, 2001). En Colombia, la Resolución 0631 de 2015 determina los rangos de vertimientos máximos permisibles de DQO (150 a $1500 \mathrm{mg} \mathrm{l}^{-1}$ ), dependiendo del sector que realiza el vertimiento, siendo los rangos más bajos los de uso doméstico y descarga en el alcantarillado público, y los más altos para la industria alimenticia y productores agropecuarios. La DQO del ASU bruta resultó en valores que varían de 600 a $1500 \mathrm{mg}^{-1}$, lo que indica una variabilidad significativa en la cantidad de MO presente en el agua servida bruta (figura 2); estos valores pueden variar por efecto de la contaminación asociada favorecida por actividades agropecuarias a pequeña escala y por el incremento en el caudal, pues en días de lluvia la carga contaminante puede bajar considerablemente.

Para el caso de la concentración de DQO en el río Ocoa $\left(429 \pm 183 \mathrm{mg} \mathrm{l}^{-1}\right)$ y la quebrada intermitente $\left(368 \pm 124 \mathrm{mg}^{-1}\right)$, los datos fueron más cercanos y se observaron procesos de contaminación asociados a los vertimientos puntuales, en los cuales su dinámica se relaciona directamente al caudal, pues en la quebrada intermitente, debido a su bajo nivel, se generaun flujo intermitente del agua; para el caso del río Ocoa, el ASU fluyó de forma más acelerada facilitando su transporte. 


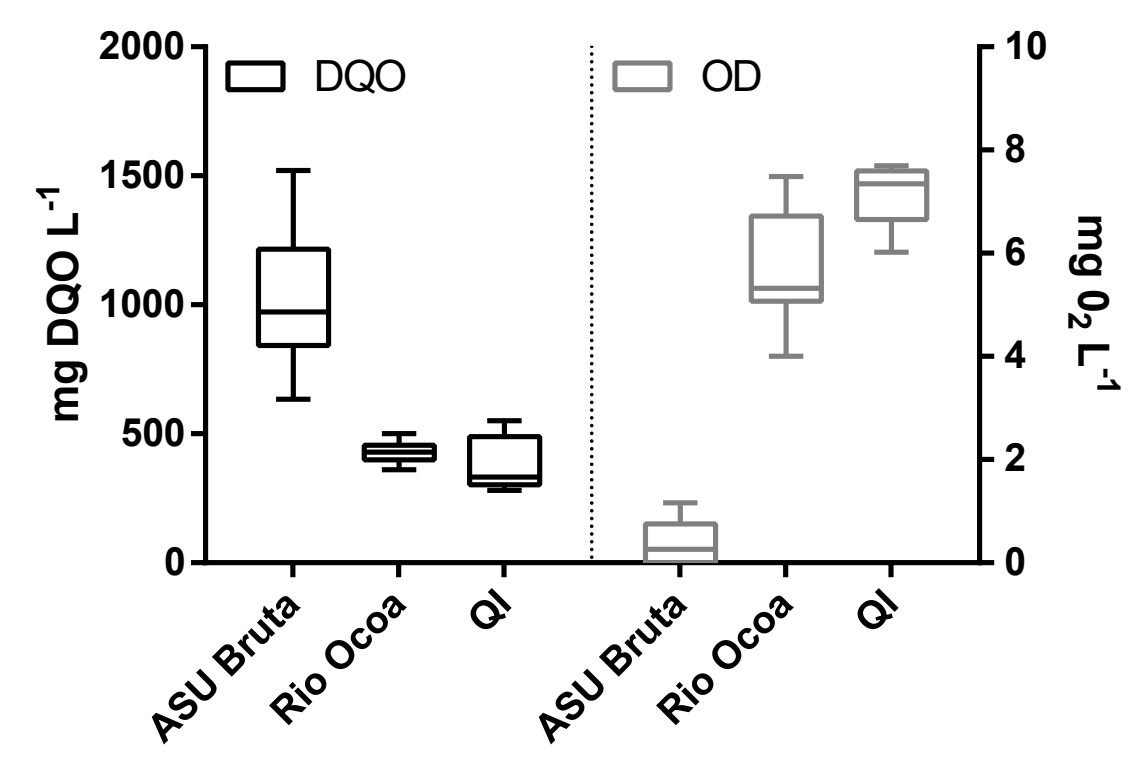

Figura 2. Box Plot para DQO y OD en el aglomerado urbano El Brillante

Fuente: elaboración propia.

Los valores de DQO presentados en ambas fuentes hídricas resultan muy elevados; sin embargo, no representan el contenido total de $\mathrm{MO}$, ya que se presentan aportes en otros puntos del cauce. A pesar de esto, se evidencian procesos de contaminación del agua en lugares de fácil acceso con fines recreativos, por lo que es necesario implementar sistemas de alcantarillado y plantas de tratamiento del ASU, así como, desarrollar actividades de gestión que permitan reducir la contaminación en el sector y capacitar a la comunidad en el uso de fuentes hídricas como estrategia de promoción de salud.

En el caso de la concentración de OD, la quebrada $\left(7,1 \pm 0,7 \mathrm{mg} \mathrm{l}^{-1}\right)$ y el río Ocoa $\left(5,7 \pm 1,8 \mathrm{mg} \mathrm{l}^{-1}\right)$ se encuentran oxigenados. Según (Arango et al., 2008), concentraciones de OD superiores a $4 \mathrm{mg} \mathrm{l}^{-1}$ para aguas naturales, pueden asegurar el correcto establecimiento de la biota acuática, por lo cual se observa que los procesos de aireación de las fuentes hídricas son favorables, y a estos niveles, se puede encontrar diversidad de especies ribereñas, lo que favorece la oxidación y eliminación de algunos de los contaminantes presentes en el agua. Por su parte, el ASU presentó valores bajos de OD $\left(0,9 \pm 0,6 \mathrm{mg}^{-1}\right)$, lo que era de esperarse debido a la elevada contaminación encontrada (Ayaz, Aktaş Akça y Findik, 2015, Oñate-Barraza y Cortez-Henao, 2020).

\section{Calidad del suelo}

Los suelos del piedemonte llanero colombiano se caracterizan por pertenecer al orden oxisol. Los parámetros fisicoquímicos se encuentran directamente relacionados con la salud del suelo y desem- 
Impacto ambiental del vertimiento de aguas servidas en aglomerados urbanos ilegales del municipio de Villavicencio, Colombia

Moyano-Salcedo., A.J. Cuadros-Segura., F.D. Pabón-Laverde., A.M. y Trujillo-Arias., J.V.

peñan un rol esencial en el desarrollo de la microbiota y mesofauna edáfica, y con esto, la cobertura vegetal que regula el sistema (Agegnehu y Amede, 2017, Gómez-Sagasti et al., 2012, Muñoz-Rojas, 2018). Estudios muestran que los suelos del lugar presentan de forma natural parámetros relevantes en el funcionamiento, entre los cuales se destacan el $\mathrm{pH}(4,2-5,5), \mathrm{CE}\left(50 \mu \mathrm{S} \mathrm{cm}{ }^{-1}\right)$, concentración de MO (1,2-1,7\%) y nitrógeno (1600 $\mathrm{mg} \mathrm{kg}^{-1}$ ) y CIC (124 meq-g $100 \mathrm{~g}^{-1}$ ) (Eurípides, Peñuela, Yacomeloy Orduz-Rodríguez, 2018, Fernandes et al., 2018,Jamioy, Menjivar y Rubiano, 2015, Nunes et al., 2017, Shibata et al., 2017). Estos parámetros pueden verse afectados fuertemente ante actividades antrópicas, como agricultura, ganadería, pastoreo, contaminación y malas prácticas, que pueden llegar a inestabilizar los procesos de regulación, generando pérdida de servicios ecosistémicos y problemáticas ambientales, lo cual afecta finalmente a la sociedad (Gómez-Sagasti et al., 2012, Castro-Garzón, Torres-Flórez y Gallardo-Lichaa, 2020).

También, se observó que las variables fisicoquímicas de los suelos se encontraban significativamente alteradas debido, tal vez, a las condiciones ambientales y decontaminación antropogénica. Se evidenció un incremento significativo del $\mathrm{pH}$ y la $\mathrm{CE}$ en comparación con los patrones normales de un oxisol de la región; en este estudio se hallaron valores hasta de 8,2 para $\mathrm{pH}$ y $517,3 \mu \mathrm{S} \mathrm{cm}^{-1}$ para $\mathrm{CE}$, así como una disminución en la concentración de nitrógeno $\left(0,03\right.$ y 0,84 $\left.\mathrm{mg} \mathrm{kg}^{-1}\right)$ y la CIC (8,2 y

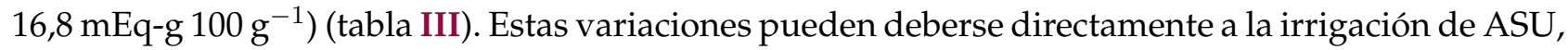
pues, al poseer cargas de diversos contaminantes, generan aportes exógenos a los sistemas, afectando las propiedades esenciales de los suelos y su buen funcionamiento (Ababsa et al., 2019, Abedi-Koupai, Mostafazadeh-Fard, Afyuni y Bagheri, 2006, Al-Othman, 2009, Becerra-Castro et al., 2015, De Oliveira et al., 2016, Zhang et al., 2018).

Algunas de las variaciones más relevantes encontradas fueron los cambios fuertes de $\mathrm{pH}$ y las altas concentraciones de CE y salinidad, lo cual puede estar afectando, en gran medida, tanto la regulación de los diferentes parámetros fisicoquímicos, como la composición edáfica y microbiana del mismo; esto ocasiona una selección de la fauna y flora por las condiciones ambientales hostiles (alta salinidad y pH básicos), y disminuye su diversidad y fertilidad considerablemente, lo cual explicaría la baja concentración de nitrógeno, puesto que su presencia en estados orgánicos y amoniacales disponibles para la vida está regulada por condiciones bióticas (Chen et al., 2014, Li et al., 2019,Zhao, Xu y Belnap, 2010).

Por otra parte, al evaluar la relación entre las variables fisicoquímicas del ASU y el suelo, se encontró un comportamiento directamente proporcional entre la $\mathrm{CE}$ y la concentración de nitrógeno con un $\mathrm{R}^{2}$ de $70,6 \%(\mathrm{p} \leq 0,01)$ así como el $\mathrm{pH}$ del suelo y la CIC con $\mathrm{R}^{2}$ de $44,6 \%(\mathrm{p} \leq 0,05)$, debido probablemente a que variables como el $\mathrm{pH}$ y la $\mathrm{CE}$ son factores que regulan de manera estricta diferentes propiedades físicas, químicas y biológicas (Kim et al., 2016, Oliver et al., 2019, Sauze et al., 2018, Tripathi et al., 2018, Zhang et al., 2020).

Por otra parte, se evidenció una relación directa entre diferentes variables fisicoquímicas del suelo en el ACP (figura 3a), el cual explica el 73 \% de los datos, donde se observa que el pH del suelo cumple un rol regulatorio sobre las diferentes variables calculadas en este estudio. Asimismo, se identificó 
Impacto ambiental del vertimiento de aguas servidas en aglomerados urbanos ilegales del municipio de Villavicencio, Colombia

Moyano-Salcedo., A.J. Cuadros-Segura., F.D. Pabón-Laverde., A.M. y Trujillo-Arias., J.V.

Tabla III. Parámetros de muestras de suelos influenciados por irrigación de ASU

\begin{tabular}{ccccccc}
\hline \multirow{2}{*}{ Muestra } & \multirow{2}{*}{$\mathbf{p H}$} & $\mathbf{C E}$ & $\mathbf{C T}$ & $\mathbf{M O}$ & $\mathbf{N}$ & $\mathbf{C I C}$ \\
\cline { 3 - 7 } & & $\mu \mathrm{Scm}^{-1}$ & $\mathbf{\%}$ & $\mathbf{\%}$ & $\mathrm{mgkb}^{-1}$ & $\mathrm{mEq}-\mathrm{g} 100 \mathrm{~g}^{-1}$ \\
\hline 2 & $8,01 \pm 0,01$ & $196 \pm 2,1$ & 5,23 & 1,79 & 0,06 & 14,7 \\
\hline 4 & $8,22 \pm 0,01$ & $249 \pm 0,01$ & 1,74 & 0,51 & 0,37 & 11,8 \\
\hline 5 & $8,18 \pm 0,01$ & $207 \pm 1,5$ & 3,51 & 1,38 & 0,28 & 16,8 \\
\hline 7 & $5,82 \pm 0,2$ & $78 \pm 1,5$ & 1,47 & 0,77 & 0,03 & 8,2 \\
\hline 9 & $8,12 \pm 0,02$ & $235 \pm 6,1$ & 3,76 & 0,76 & 0,03 & 11,2 \\
\hline 11 & $7,94 \pm 0,01$ & $226 \pm 0,6$ & 3,18 & 1,28 & 0,25 & 14,8 \\
\hline 12 & $7,69 \pm 0,01$ & $165 \pm 0,6$ & 4,14 & 2,06 & 0,28 & 10 \\
\hline 14 & $7,93 \pm 0,01$ & $517 \pm 3,1$ & 3,12 & 1,54 & 0,84 & 10,7 \\
\hline 15 & $6,63 \pm 0,02$ & $58 \pm 0,6$ & 3,52 & 1,61 & 0,17 & 9,9 \\
\hline
\end{tabular}

Fuente: elaboración propia.

una relación estrecha entre las concentraciones de CT y MO con comportamientos directamente proporcionales y coherentes con las demás variables calculadas en el suelo. Además, se observa que el ACP para las variables del ASU (figura 3b) representa el 61,4\% de los datos, donde existe una relación relevante entre variables como $\mathrm{CE}, \mathrm{OD}, \mathrm{DQO}$ y $\mathrm{pH}$, siendo el OD y el $\mathrm{pH}$ los que influencian de manera más significativa el comportamiento del sistema.

Finalmente, con el fin de evaluar la relación y determinar el posible efecto que generan la constante irrigación por ASU en suelos del sector, se diseñó un ACP de todas las variables (figura 3c), el cual representó el 55,6 \% de los datos, de lo cual se determinó que las variables más relevantes fueron el OD y la turbiedad del agua.

Se identificó una relación entre dos grupos diferentes de variables: un comportamiento directo entre el pH del agua y la DQO con variables del suelo como la CIC y las concentraciones de CT y $\mathrm{MO}$, y propiedades de turbiedad y color del agua (influenciadas a su vez por la concentración del lodo, SSV y ST) sobre el pH, la CE y la concentración de nitrógeno del suelo. Así, resulta notaria la importancia del $\mathrm{pH}$ como regulador de los procesos 0,50 químicos (oxidorreducción) en el agua y el suelo, y determinante en la conversión de contaminantes y el ciclo del nitrógeno. Por su parte, la DQO representa el aporte de MO que puede existir debido a los vertimientos de ASU, lo que representa uno de los principales parámetros a controlar para asegurar condiciones ambientales favorables para el sostenimiento de las especies de fauna, flora y el desarrollo de cultivos.

Por último, al evaluar la concentración de fósforo disponible y elementos traza presentes en el suelo, se encontró que los valores (tabla IV) están en los rangos establecidos por investigaciones 
Impacto ambiental del vertimiento de aguas servidas en aglomerados urbanos ilegales del municipio de Villavicencio, Colombia

Moyano-Salcedo., A.J. Cuadros-Segura., F.D. Pabón-Laverde., A.M. y Trujillo-Arias., J.V.
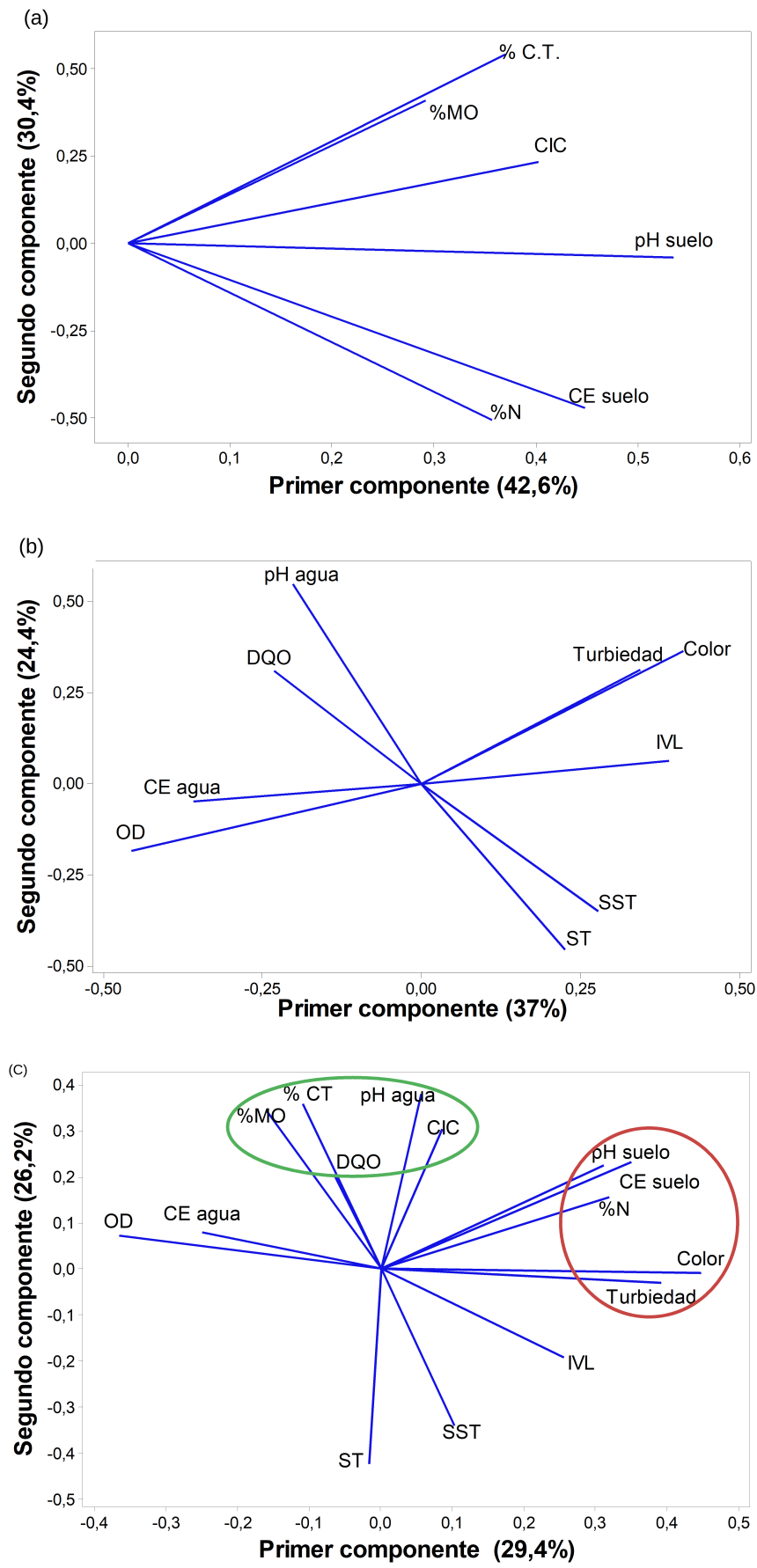

Figura 3. Análisis de componentes principales (ACP) de los diferentes parámetros fisicoquímicos (a) ACP de parámetros fisicoquímicos de suelo. (b) ACP de parámetros fisicoquímicos de ASU. (c) ACP del conjunto de parámetros fisicoquímicos.

Fuente: elaboración propia 
Impacto ambiental del vertimiento de aguas servidas en aglomerados urbanos ilegales del municipio de Villavicencio,

realizadas en suelos oxisólicos del lugar (Eurípides, Peñuela, Yacomeloy Orduz-Rodríguez, 2018, Fernandes et al., 2018). Sin embargo, hubo un incremento en la concentración de fósforo disponible (56 $\left.\mathrm{mg} \mathrm{kg}^{-1}\right)$, así como elementos traza como el As $\left(4 \mathrm{mg} \mathrm{kg}^{-1}\right)$, los cuales no representan ningún daño ni perjuicio para el normal funcionamiento del suelo (Fernandes et al., 2018, Rueda, Rodríguez y Madriñán, 2011,Mahecha-Pulido, Trujillo-González y Torres-Mora, 2015,Nunes et al., 2017).

Tabla IV. Concentración de fósforo disponible y perfil de elementos traza en la muestra de suelo $2(0-20 \mathrm{~cm})$ en unidades de $\mathrm{mg} \mathrm{kg}^{-1}$

\begin{tabular}{cccccc}
\hline Traza & Concentración & Traza & Concentración & Traza & Concentración \\
\hline Fósforo disponible & 56 & $\mathrm{Cu}$ & 8 & $\mathrm{Ni}$ & 5 \\
\hline $\mathrm{Al}$ & 3440 & $\mathrm{Cr}$ & 12 & $\mathrm{~Pb}$ & 10 \\
\hline $\mathrm{As}$ & 3 & $\mathrm{Sr}$ & 5 & $\mathrm{~V}$ & 8 \\
\hline $\mathrm{Ba}$ & 20 & $\mathrm{Li}$ & 7 & $\mathrm{Zn}$ & 23 \\
\hline $\mathrm{B}$ & 4 & $\mathrm{Mn}$ & 122 & & \\
\hline
\end{tabular}

Fuente: elaboración propia.

\section{CONCLUSIONES}

La alta contaminación por MO hacia el río Ocoa y la DQO media en el ASD presentan valores significativamente elevados, típicos de aguas vertidas por explotaciones agropecuarias o industrias alimenticias; por tanto, es probable que los vertimientos en este estudio tengan aportes de este tipo de actividades, lo cual, supone una alteración negativa de la calidad a la fuente receptora y pérdida de la biota acuática. Esto puede estar modelado también por la hidrodinámica de la corriente o aspectos fisográficos del sector que generan variaciones en propiedades como el OD del agua, el cual puede favorecer, en algún momento, los procesos de depuración del sistma.

Se observó un efecto directo de los vertimientos de ASD en los suelos, lo que afecta los parámetros fisicoquímicos y los factores biológicos como la cobertura vegetal, meso- y microfauna, por lo cual se evidencian procesos de pérdidas de fertilidad y acumulación de MO no degradada, generando problemas de salud pública.

Por esta razón es importante la gestión del agua en el lugar, mediante la implementación de sistemas de saneamiento básico adecuados (alcantarillado o recolección periódica de residuos sólidos); el tratamiento del agua residual de forma unifamiliar (sistema alternativo: pretratamiento, pozo séptico y filtración terciaria) o colectiva (planta de tratamiento convencional -PTAR-), y el control y asistencia técnica sostenible de los potenciales emprendimientos agropecuarios en el sector (por ejemplo, manuales de buenas prácticas agropecuarias, uso eficiente del agua, sistemas de tratamiento, etc.). 
Por último, el conjunto de esas medidas de gestión, que deben ser impulsadas por el gobierno municipal e implementadas con la participación de la comunidad local, permitiría reducir la generación y propagación de enfermedades, y promover la calidad ambiental y de vida.

\section{FINANCIAMIENTO}

El presente estudio fue financiado y avalado por la Universidad Santo Tomás, sede Villavicencio.

\section{REFERENCIAS}

[Ababsa et al., 2019] Ababsa, N., Kribaa, M., Tamrabet, L., Addad, D., Hallaire, V. y Ouldjaoui, A. (2019). Long- term effects of wastewater reuse on hydro physicals characteristics of grassland grown soil in semi-arid Algeria. Journal of King Saud University - Science, 32(1). https: //doi.org/10.1016/j.jksus.2019.09.007个Ver página 52

[Abedi-Koupai, Mostafazadeh-Fard, Afyuni y Bagheri, 2006] Abedi-Koupai, J., Mostafazadeh-Fard, B., Afyuni, M. y Bagheri, M. R. (2006). Effect of treated wastewater on soil chemical and physical. Plant and Soil Environment Soil Environment, 52(8), 335-344. https://doi .org/10.17221/ $3450-$ P SE $\uparrow$ Ver página 52

[Agegnehu y Amede, 2017] Agegnehu, G. y Amede, T. (agosto de 2017). Integrated soil fertility and plant nutrient management in tropical agro-ecosystems: a review. Pedosphere, 27, 662-680. DOI: https://doi.org/10.1016/S1002-0160(17)60382-5个Ver página 52

[Al-Othman, 2009] Al-Othman, A. A. (2009). Effect of treated domestic wastewater on physical and chemical characteristics of soils. Journal of Applied Sciences, 9, 901-908. DOI: https://doi . org/10.3923/jas.2009.901.908 个Ver página 52

[Amin y Flowers, 2004] Amin, M. y Flowers, T. (2004). Evaluation of Kjehdahl digestion method. Journal of Research, 15(2), 159-179. $\uparrow$ Ver página 48

[Arango et al., 2008] Arango M., Álvarez L., Arango G., Torres O. y Monsalve A. (2008). Calidad del agua de las quebradas la cristalina y la risaralda San Luis, Antioquia. Revista EIA, 9, 121-141. $\uparrow$ Ver página 51

[Ayaz, Aktaş Akça y Findik, 2015] Ayaz, S. T., Aktaş, Ö., Akça, L. y Findik, N. (2015). Effluent quality and reuse potential of domestic wastewater treated in a pilot-scale hybrid constructed wetland system. Journal of Environmental Management, 156, 115-120. DOI: https://doi .org/10.1016/ j.jenvman.2015.03.042 个Ver página 49,51 
Impacto ambiental del vertimiento de aguas servidas en aglomerados urbanos ilegales del municipio de Villavicencio, Colombia

Moyano-Salcedo., A.J. Cuadros-Segura., F.D. Pabón-Laverde., A.M. y Trujillo-Arias., J.V.

[Becerra-Castro et al., 2015] Becerra-Castro, C., Lopes, A. R., Vaz-Moreira, I., Silva, E. F., Manaia, C. M. y Nunes, O. C. (2015). Wastewater reuse in irrigation: a microbiological perspective on implications in soil fertility and human and environmental health. Environment International, 75, 117-135. DOI: https://doi.org/10.1016/j.envint.2014.11.001个Ver página 52

[Bradstreet, 1954] Bradstreet, R. B. (1954). Kjeldahl method for organic nitrogen. Analytical Chemistry, 26(1), 185-187. DOI: https://doi.org/10.1021/ac60085a028 个Ver página 48

[Briceño, 2015] Briceño, G. (2015). Evolución de la integridad estructural de ecosistemas lóticos del piedemonte llanero frente a la intervención antrópica. Acta Biologica Colombiana, 20(2), 133-144. DOI: https://doi.org/10.15446/abc.v20n2.42307个Ver página 46

[Cantanhede, Monge, Sandoval y Caycho, 2005] Cantanhede, A., Monge, G., Sandoval, L. y Caycho, C. (2005). Procedimientos estadísticos para los estudios de caracterisacion de residuos solidos. AIDS, Ingeniería y Ciencias Ambientales: Investigación, Desarrollo y Práctica, 1, 1-14. $\uparrow$ Ver página 46

[Castro-Garzón, Torres-Flórez y Gallardo-Lichaa, 2020] Castro-Garzón, H., Torres-Flórez, D. y Gallardo-Lichaa, N. J. (2020). Análisis de la intervención antrópica en cuerpos de agua: caso caño Banderas, en el municipio Puerto López (Meta, Colombia). Tecnura, 24(65) 77-84.

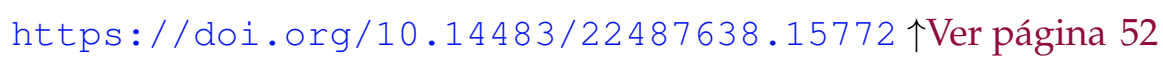

[Cecconet, Molognoni, Callegari y Capodaglio, 2018] Cecconet, D., Molognoni, D., Callegari, A. y Capodaglio, A. G. (2018). Agro-food industry wastewater treatment with microbial fuel cells: energetic recovery issues. International Journal of Hydrogen Energy, https : / doi . org/10.1016/j . ijhydene.2017.07.231 $\uparrow$ Ver página 50

[Chen et al., 2014] Chen, X., Song, B., Yao, Y., Wu, H., Hu, J. y Zhao, L. (2014). Aromatic plants play an important role in promoting soil biological activity related to nitrogen cycling in an orchard ecosystem. Science of the Total Environment, 472, 939-946. DOI: https: / / doi . org/10.1016/j. scitotenv.2013.11.117 ^er página 52

[De Oliveira et al., 2016] De Oliveira, P. C. P., Gloaguen, T. V., Gonçalves, R. A. B., Santos, D. L. y Couto, C. F. (2016). Soil chemistry after irrigation with treated wastewater in semiarid climate. Revista Brasileira de Ciencia Do Solo, 40, 1-13. DOI: https://doi.org/10.1590/ 18069657 rbcs20140664 个Ver página 52

[Enang, Yerima, Kome y Van Ranst, 2018] Enang, R. K., Yerima, B. P. K., Kome, G. K. y Van Ranst, E. (2018). Assessing the effectiveness of the Walkley-Black method for soil organic carbon determination in tephra soils of cameroon. Communications in Soil Science and Plant Analysis, 49(19), 2379-2386. DOI: https://doi.org/10.1080/00103624.2018.1510948 个er página 48 
Impacto ambiental del vertimiento de aguas servidas en aglomerados urbanos ilegales del municipio de Villavicencio, Colombia

Moyano-Salcedo., A.J. Cuadros-Segura., F.D. Pabón-Laverde., A.M. y Trujillo-Arias., J.V.

[Eurípides, Peñuela, Yacomeloy Orduz-Rodríguez, 2018] Eurípides, J., Peñuela, B., Yacomelo, M. y Orduz-Rodríguez, J. (2018). Efecto del yeso sobre las características químicas de un Oxisol de la Orinoquia colombiana cultivado con lima ácida. Temas Agrarios, 23(2), 154-163. https://doi . org/10.21897/rta.v23i2.1299 Ter página 52, 55

[Fernandes et al., 2018] Fernandes, J., Chaves, L., Mendes, J., Chaves, I. de B. y Tito, G. (2018). Soil chemical amendments and the macronutrients mobility evaluation in oxisol treated with biochar. Journal of Agricultural Science, 10(10), 238. DOI: https://doi.org/10.5539/jas. v10n10p238 $\uparrow$ Ver página 52, 55

[Gómez-Sagasti et al., 2012] Gómez-Sagasti, M., Alkorta, I., Becerril, J., Epelde, L., Anza, M. y Garbisu, C. (2012). Microbial monitoring of the recovery of soil quality during heavy metal phytoremediation. Water, Air, and Soil Pollution, 223(6), 3249-3262. DOI: https://doi .org/10.1007/ s11270-012-1106-8 $\uparrow$ Ver página 52

[Hernández-Gómez, Rojas-Robles y Sánchez-Calderón, 2013] Hernández-Gómez, A., Rojas-Robles, R. y Sánchez-Calderón., F. (2013). Cambios en el uso del suelo asociados a la expansión urbana y la planeación en el corregimiento de Pasquilla, zona rural de Bogotá (Colombia). Cuadernos de Geografía - Revista Colombiana de Geografía., 22, 257-271. Recuperado de https : / www . redalyc . org/pdf/2818/281826970012.pdf 个Ver página 45

[Hernández y Sánchez, 2015] Hernández, D. y Sánchez, S. (2015). Diseño de una planta de tratamiento de agua residual para el municipio de San Marcos, departamento de Sucre. [Trabajo de grado]. Facultad de Ingeniería, Universidad Católica de Colombia. Bogotá, Colombia. Recuperado de https : //repository.ucatolica.edu.co/bitstream/10983/2395/3/Artículo.pdf 个Ver página 50

[Ideam, 2015] Instituto de Hidrología, Meteorología y Estudios Ambientales (Ideam) (2015). Estudio Nacional del Agua 2014. Bogotá. $\uparrow$ Ver página 46

[IGAC, 2006] Instituto Geográfico Agustín Codazzi (IGAC) (2006). Métodos analíticos del laboratorio de suelos. Bogotá, Colombia. $\uparrow$ Ver página 46

[Jamioy, Menjivar y Rubiano, 2015] Jamioy, D., Menjivar, J. y Rubiano, Y. (2015). Indicadores químicos de calidad de suelos en sistemas productivos del piedemonte de los Llanos Orientales de Colombia. Acta Agronómica, 64(4), 302-307. DOI: https : / / doi . org/10 . 15446 /acag •v64n 4 . $38731 \uparrow$ Ver página 52

[Kim et al., 2016] Kim, J., Roh, A., Choi, S., Kim, E., Choi, M., Ahn, B., .. Weon, H. Y. (2016). Soil $\mathrm{pH}$ and electrical conductivity are key edaphic factors shaping bacterial communities of greenhouse soils in Korea. Journal of Microbiology, 54(12), 838-845. DOI https : / / doi . org/10 . 1007 / s12275-016-6526-5 个Ver página 52 
Impacto ambiental del vertimiento de aguas servidas en aglomerados urbanos ilegales del municipio de Villavicencio, Colombia

Moyano-Salcedo., A.J. Cuadros-Segura., F.D. Pabón-Laverde., A.M. y Trujillo-Arias., J.V.

[Lampoglia, Agüero y Barrios, 2008] Lampoglia, T., Agüero, R. y Barrios, C. (2008). Orientaciones para agua y saneamiento en zonas rurales. Organización Panamericana de la Salud. Recuperado de https://n9.cl/ersos $\uparrow$ Ver página 45

[Li et al., 2019] Li, S., Peng, C., Cheng, T., Wang, C., Guo, L. y Li, D. (2019). Nitrogen-cycling microbial community functional potential and enzyme activities in cultured biofilms with response to inorganic nitrogen availability. Journal of Environmental Sciences, 76, 89-99. DOI: https: //doi.org/10.1016/j.jes.2018.03.029个Ver página 52

[Londoño et al., 2002] Londoño, M., Osorno, M., Vélez, J., García, F., Montoya, M. y Olaya, U. (2002). Banatura: programa de gestión social y ambiental del sector bananero colombiano. En Memorias

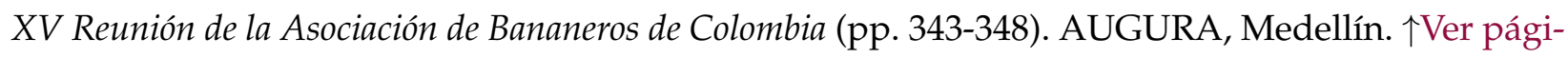
na 45

[Madsen, Lévêque, Omiste y Miyagi, 2018] Madsen, L., Lévêque, C., Omiste, J. y Miyagi, H. (2018). Chapter 11: Time-dependent restricted-active-space self-consistent-field theory for electron dynamics on the attosecond timescale. En M. J. J. Vrakking y F. Lepine (eds.), Attosecond Molecular Dynamics (pp. 386-423). Cambridge, Reino Unido: Royas Society of Chemistry. DOI: https: //doi.org/10.1039/9781788012669-00386 †er página 49,50

[Mahecha-Pulido, Trujillo-González y Torres-Mora, 2015] Mahecha-Pulido, J., Trujillo-González, J. M. y Torres-Mora, M. A. (2015). Contenido de metales pesados en suelos agrícolas de la región del Ariari, departamento del Meta. Orinoquia, 19(1), 118-122. https://doi.org/10.22579/ $20112629.345 \uparrow$ Ver página 55

[Makara y Kowalski, 2015] Makara, A. y Kowalski, Z. (2015). Pig manure treatment and purification by filtration. Journal of Environmental Management, 161, 317-324. DOI: https://doi.org/10 . $1016 / j \cdot j e n v m a n .2015 .07 .022 \uparrow$ Ver página 50

[Miyazawa et al., 2000] Miyazawa, M., Pavan, M., De Oliveira, E., Ionashiro, M. y Silva, A. (2000). Gravimetric determination of soil organic matter. Brazilian Archives of Biology and Technology, 43(5), 475-478. DOI: https://doi.org/10.1590/s1516-89132000000500005 个Ver página 48

[Muñoz-Rojas, 2018] Muñoz-Rojas, M. (2018). Soil quality indicators: critical tools in ecosystem restoration. Current Opinion in Environmental Science \& Health, 5, 47-52. DOI: https: / doi .org/ $10.1016 / j$.coesh.2018.04.007 个Ver página 52

[Nunes et al., 2017] Nunes, M., Vaz, C., Denardin, J., Van Es, H., Libardi, P. y da Silva, A. (2017). Physicochemical and structural properties of an oxisol under the addition of straw and lime. Soil Science Society of America Journal, 81(6), 1328-1339. DOI: https: // doi .org/10.2136/sssaj2017. $07.0218 \uparrow$ Ver página 52, 55 
Impacto ambiental del vertimiento de aguas servidas en aglomerados urbanos ilegales del municipio de Villavicencio, Colombia

Moyano-Salcedo., A.J. Cuadros-Segura., F.D. Pabón-Laverde., A.M. y Trujillo-Arias., J.V.

[Oliver et al., 2019] Oliver, D., Li, Y., Orr, R., Nelson, P., Barnes, M., McLaughlin, M. y Kookana, R. (2019). The role of surface charge and $\mathrm{pH}$ changes in tropical soils on sorption behaviour of perand polyfluoroalkyl substances (PFASs). Science of the Total Environment, 673, 197-206. DOI: https : //doi.org/10.1016/j.scitotenv.2019.04.055个Ver página 52

[Oñate-Barraza y Cortez-Henao, 2020] Oñate-Barraza., H. C. y Cortez-Henao, G. Y.(2020). Estado del agua del río Cesar por vertimientos residuales de la ciudad de Valledupar. Bioindicación por índice BMWP/Col. Tecnura, 24(65), 39-48. DOI: https://doi .org/10.14483/22487638.15766 $\uparrow$ Ver página 51

[Pérez, 2001] Pérez R. (2001). Porcicultura y contaminacion del agua en La Piedad, Michoacán, México. Revista Internacional de Contaminación Ambiental, 17(1), 5-13. Recuerado de https: / / n 9 . cl/ skd49 9 Ver página 50

[Rice, Baird e Eaton, 2017] Rice, E. W., Baird, R. B. e Eaton, A. D. (eds.). (2017). Standard methods for examination of water and wastewater. Denver, EE. UU.: American Water Works Association (AWWA). 个Ver página 47

[Rodríguez-Miranda, García-Ubaque y Pardo-Pinzón, 2015] Rodríguez-Miranda, J. P., GarcíaUbaque, C. A. y Pardo-Pinzón, J. (2015). Selección de tecnologías para el tratamiento de aguas residuales municipales. Tecnura, 19(46), 149-164. DOI: https://doi.org/10.14483/ udistrital.jour.tecnura.2015.4.a12 个Ver página 45

[Rueda, Rodríguez y Madriñán, 2011] Rueda, G., Rodríguez, J. y Madriñán, R. (2011). Metodologías para establecer valores de referencia de metales pesados en suelos agrícolas perspectivas para Colombia. Acta Agronómica, 60(3), 203-217. $\uparrow$ Ver página 55

[Sadzawka et al., 2006] Sadzawka, A., Carrasco, M., Grez, R., Mora, M., Flores, H. y Neaman A. (2006). Metodos de análisis recomendados para los suelos de Chile, revisión 2006. Santiago de Chile, Chile: Instituto de Investigaciones Agropecuarias. $\uparrow$ Ver página 47

[Samboni, Aldemar y Yesid, 2011] Samboni, N., Aldemar, R. y Yesid, C. (2011). Aplicación de los indicadores de calidad y contaminación del agua en la determinación de la oferta hídrica neta. Environmental Engineering, 60(2), 49-60. $\uparrow$ Ver página 49

[Sarkar, Chakrabarti, Vijaykumary Kale, 2006] Sarkar, B., Chakrabarti, P., Vijaykumar, A. y Kale, V. (2006). Wastewater treatment in dairy industries - possibility of reuse. Desalination, 195(1-3), 141152. DOI: https://doi.org/10.1016/j.desal.2005.11.015个Ver página 50

[Sauze et al., 2018] Sauze, J., Jones, S., Wingate, L., Wohl, S. y Ogée, J. (2018). The role of soil pH on soil carbonic anhydrase activity. Biogeosciences, 15(2), 597-612. DOI: https:// doi . org/10 . 5194/bg-15-597-2018 个Ver página 52 
Impacto ambiental del vertimiento de aguas servidas en aglomerados urbanos ilegales del municipio de Villavicencio, Colombia

Moyano-Salcedo., A.J. Cuadros-Segura., F.D. Pabón-Laverde., A.M. y Trujillo-Arias., J.V.

[Shibata et al., 2017] Shibata, M., Sugihara, S., Mvondo-Ze, A., Araki, S. y Funakawa, S. (2017). Nitrogen flux patterns through oxisols and ultisols in tropical forests of Cameroon, Central Africa. Soil Science and Plant Nutrition, 63(3), 306-317. DOI: https: / / doi . org/10.1080/00380768. 2017.1341285 ^Ver página 52

[Stevenson, Ramírez, Casas y Henao-Díaz, 2017] Stevenson, P., Ramírez, M., Casas, L. y Henao-Díaz, F. (2017). Forestación de bosques en sabanas de la altillanura colombiana: relevancia de las condiciones ambientales para el establecimiento de plántulas. Biota Colombiana, 18(1), 99-118. https: //doi.org/10.21068/c2017v18s01a06 †er página 46

[Subtil, Moyano y Bez, 2018] Subtil, E., Moyano, A. y Bez, T. (2018). Remoção de nitrogênio e matéria orgânica em diferente concentração de oxigênio dissolvido em um biorreator de membrana submersa com biofilme. Conferencia en XIV Simposio Ítalo-Brasileiro de Engenharia Sanitária e Ambiental. 18-20 de junio. Associação Brasileira de Engenharia Sanitária e Ambiental. Foz do Iguaçu. Ver página 45,49

[Torres et al., 2010] Torres, P., Cruz, C., Patiño, P., Escobar, J. y Pérez, A. (2010). Applying water quality indexes (WQI) to the use of water sources for human consumption. Ingeniería e Investigación, 30(3), 86-95. $\uparrow$ Ver página 49

[Tripathi et al., 2018] Tripathi, B., Stegen, J., Kim, M., Dong, K., Adams, J. y Lee, Y. (2018). Soil pH mediates the balance between stochastic and deterministic assembly of bacteria. ISME Journal, 12(4), 1072-1083. DOI: https: / / doi .org/10.1038/s41396-018-0082-4 个Ver página 52

[USDA, 2014] United States Department of Agriculture (USDA) (2014). Soil survey field and laboratory methods manual. DOI: https://doi.org/10.13140/RG.2.1.3803.8889个Ver página 47

[US EPA, 1994] United States Environmental Protection Agency (US EPA) (1994). Method 200.7: Determination of metals and trace elements in water and wastes by inductively coupled plasma-atomic emission spectrometry. Revision 4.4. Cincinnati, EE. UU. $\uparrow$ Ver página 48

[US EPA, 2007] United States Environmental Protection Agency (US EPA) (2007). Method 3015A (SW846): Microwave assisted acid digestion of aqueous samples and extracts. Revision 1. Washington, D. C., EE. UU. $\uparrow$ Ver página 48

[Zhang et al., 2018] Zhang, Y., Fu, C., Liu, X., Li, X., Jing, Q., Wei, X., .. Yan, P. (2018). Effect of poultry wastewater irrigation on nitrogen, phosphorus and carbon contents in farmland soil. Open Chemistry, 16(1), 968-977. DOI: https: / / doi .org/10.1515/chem-2018-0111 个Ver página 52

[Zhang et al., 2020] Zhang, Y., Dai, S., Huang, X., Zhao, Y., Zhao, J., Cheng, Y., ... Zhang, J. (2020). $\mathrm{pH}$-induced changes in fungal abundance and composition affects soil heterotrophic nitrification after 30 days of artificial pH manipulation. Geoderma, 366, 114255. DOI: https : / / doi . org/ 10 . $1016 / j$.geoderma.2020.114255 个Ver página 52 
Impacto ambiental del vertimiento de aguas servidas en aglomerados urbanos ilegales del municipio de Villavicencio, Colombia

Moyano-Salcedo., A.J. Cuadros-Segura., F.D. Pabón-Laverde., A.M. y Trujillo-Arias., J.V.

[Zhao, Xu y Belnap, 2010] Zhao, Y., Xu, M. y Belnap, J. (2010). Potential nitrogen fixation activity of different aged biological soil crusts from rehabilitated grasslands of the hilly Loess Plateau, China. Journal of Arid Environments, https://doi.org/10.1016/j.jaridenv.2010.04.006 Ver página 52 